\title{
Memory Loss and Frontal Cognitive Dysfunction in a Patient with Adult-onset Neuronal Intranuclear Inclusion Disease
}

\author{
Kunihiko Araki, Jun Sone, Yusuke Fujioka, Michihito Masuda, Reiko Ohdake, \\ Yasuhiro Tanaka, Tomohiko Nakamura, Hirohisa Watanabe and Gen Sobue
}

\begin{abstract}
Neuronal intranuclear inclusion disease (NIID) is an uncommon progressive neurodegenerative disorder. Adult-onset NIID can result in prominent dementia. We herein describe the case of a 74-year-old man who presented with dementia, cerebellar ataxia, neuropathy, and autonomic dysfunction. Diffusion-weighted imaging showed hyperintensity of the corticomedullary junction. Fluid-attenuated inversion recovery images showed frontal-dominant white matter hyperintensity. NIID was diagnosed from the presence of intranuclear inclusions in a skin biopsy sample. Neuropsychological testing revealed memory loss and frontal cognitive dysfunction, especially in relation to language and executive functions. We were therefore able to confirm the association of NIID with cognitive dysfunction.
\end{abstract}

Key words: executive function, leukoencephalopathy, memory loss, neuronal intranuclear inclusion disease, skin biopsy

(Intern Med 55: 2281-2284, 2016)

(DOI: 10.2169/internalmedicine.55.5544)

\section{Introduction}

Neuronal intranuclear inclusion disease (NIID) is an uncommon progressive neurodegenerative disorder characterized by eosinophilic hyaline intranuclear inclusions in nearly all types of central, peripheral, and autonomic neurons (1). NIID is clinically heterogeneous and may cause symptoms such as cerebellar ataxia, dementia, pyramidal and extrapyramidal signs, generalized convulsion, isolated resting tremor, and autonomic dysfunction (2-18). We previously showed that the detection of intranuclear inclusions in dermal cells was a reliable diagnostic test for NIID (16). In addition, we identified a characteristic finding of a high signal intensity in the corticomedullary junction on diffusionweighted imaging (DWI) in NIID patients with leukoencephalopathy diagnosed by a skin biopsy (18).

The signs of dementia have been previously described in adult-onset NIID patients $(3,6,9,12,18)$. However, objective the neuropsychological assessments of such signs have so far been limited because, until the recent advent of a diagnosis by a skin biopsy (16), the clinical signs and symptoms of dementia in these patients had been retrospectively investigated only after a diagnosis by an autopsy. We herein report the case of a patient with adult-onset NIID who was diagnosed with progressive memory loss and frontal cognitive dysfunction.

\section{Case Report}

A 74-year-old man was referred to our hospital with a 7year history of memory loss and a 3-year history of gait disturbance. After retiring, he took a walk every day for 7 years. However, 5 years previously he had begun to lose his way. He had spent the past several years only at home, during which time he had often mistaken the names of his daughter and grandchildren. No hyperoral or stereotypic behaviors, poor hygiene, or impulsive acts representative of frontotemporal dementia were observed. He had a past medical history of hypertension, asthma, and benign 

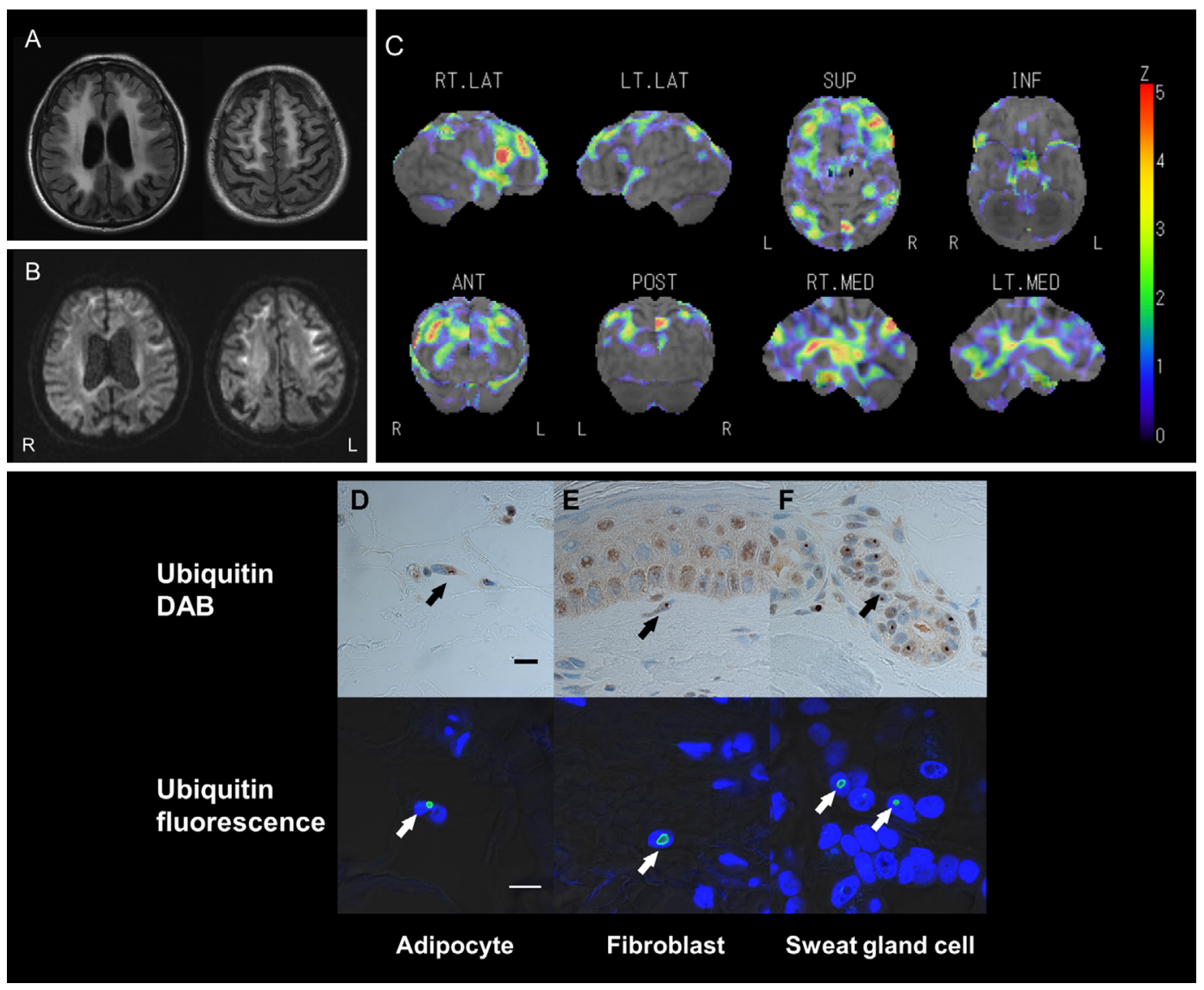

Figure. Fluid-attenuated inversion recovery (FLAIR) images, diffusion-weighted imaging (DWI), single photon emission computed tomography (SPECT), and histopathological features of neuronal intranuclear inclusions in dermal cells. A: FLAIR images show frontal-dominant white matter hyperintensity with diffuse brain atrophy. B: DWI shows hyperintensity of the corticomedullary junction. C: A three-dimensional stereotactic surface projection of SPECT with $\left[{ }^{123}\right.$ I]iodoamphetamine shows hypoperfusion in the fronto-parietal regions. D to F: Immunohistological study of skin biopsy samples with anti-ubiquitin antibody. (D) adipocytes, (E) fibroblasts, and (F) sweat gland cells. The upper row demonstrates immunostaining with 3,3'-diaminobenzidine (DAB), whereas the lower row shows immunofluorescence staining with 4',6-diamidino-2-phenylindole (DAPI) (blue) and ubiquitin (green). (Scale bars $=10 \mu \mathrm{m}$ ). In both studies, intranuclear inclusions were detected and positively stained with anti-ubiquitin antibody in adipocytes, fibroblasts, and sweat gland cells.

prostatic hyperplasia, with no family history of dementia. His cranial nerve functions were intact except for bilateral miosis of less than $2.0 \mathrm{~mm}$ (1.7 on the right and $1.9 \mathrm{~mm}$ on the left) and dysarthria. Manual muscle testing was normal, although hyporeflexia and hypopallesthesia in the upper and lower limbs were observed. The patient had a wide-based gait, predominantly left-sided limb ataxia, and bilateral dysdiadochokinesia. He was using clean intermittent catheterization because he could not urinate, although he was able to spontaneously urinate with silodosin treatment $(4 \mathrm{mg}$, once daily). Laboratory examinations revealed normal levels of thiamine, vitamin $\mathrm{B}_{12}$, thyroid hormone, and prostate-specific antigen. A cerebrospinal fluid examination showed an opening pressure of $9 \mathrm{~cm} \mathrm{H}_{2} \mathrm{O}$, leukocyte count of 14 cells $/ \mu \mathrm{L}$, total protein level of $76 \mathrm{mg} / \mathrm{dL}$, and glucose level of $58 \mathrm{mg} /$ dL. A nerve conduction study revealed slightly reduced motor conduction velocities of 44, 44, and $36 \mathrm{~m} / \mathrm{s}$ in the right median, ulnar, and tibial nerves, respectively, and almost normal sensory conduction velocities of 50,48 , and $40 \mathrm{~m} / \mathrm{s}$ in the right median, ulnar, and sural nerves, respectively. Magnetic resonance imaging (MRI) demonstrated frontaldominant white matter hyperintensity on fluid-attenuated inversion recovery (FLAIR) images, with diffuse brain atrophy (Figure A), and hyperintensity of the corticomedullary junction on DWI (Figure B). Single photon-emission computed tomography (SPECT) of the head with $\left[{ }^{123} \mathrm{I}\right]$ iodoamphetamine showed hypoperfusion in the fronto-parietal regions (Figure C). $\left[{ }^{123} \mathrm{I}\right]$ metaiodobenzylguanidine myocardial scintigraphy was normal according to the criteria at our center, with an early heart-to-mediastinum (H/M) ratio of 3.38 and a delayed H/M ratio of 4.41. Initially, electronic uroflowmetry showed a large post-void residual urine volume (PVR= $250 \mathrm{~mL}$; normal range 50 to $100 \mathrm{~mL}$ ). When the patient was able to urinate after taking $4 \mathrm{mg}$ silodosin, uroflowmetry showed reductions in the maximum urinary flow rate $\left(\mathrm{Q}_{\max }=\right.$ $3.3 \mathrm{~mL} / \mathrm{s}$; normal rate $<10 \mathrm{~mL} / \mathrm{s}$ ) and voided volume ( $\mathrm{VV}=$ 
$62.3 \mathrm{~mL})$, as well as an improvement in the PVR $(40 \mathrm{~mL})$. These results were suggestive of a bladder outlet obstruction due to neurogenic bladder dysfunction. Orthostatic hypotension was seen in the head-up tilt test, with a decrement of $43 \mathrm{mmHg}$ in systolic blood pressure. During this test, the level of plasma noradrenaline rose from 0.229 to $0.542 \mathrm{ng} /$ $\mathrm{mL}$. The coefficient of variation of the R-R interval on electrocardiograms was normal $(2.31 \%)$. During phase IV of the Valsalva maneuver, the systolic blood pressure did not increase, despite a normal heart rate response, perhaps due to the dysfunction of the efferent sympathetic system. A skin biopsy performed on the right leg demonstrated ubiquitinpositive intranuclear inclusions among adipocytes, fibroblasts, and sweat gland cells (Figure D to F). The patient did not carry the fragile $X$ mental retardation1 premutation.

Neuropsychological testing of the patient revealed memory loss, visuospatial dysfunction, and frontal cognitive dysfunction, especially in language and executive functions. His Mini-Mental State Examination (MMSE) score of 21/30 indicated disorientation (3/5), normal delayed recall (3/3), loss of numerical calculation ability (1/5), impaired repetition (0/ 1 ), and poor visuospatial function (0/1). His score of $59 / 100$ on Addenbrooke's Cognitive Examination Revised (ACE-R) indicated disorientation (2/5); loss of delayed recall (1/7); anterograde (4/7) and retrograde (2/4) amnesia; markedly impaired verbal fluency (phonemic, 0/7; semantic, 0/7); impaired naming $(9 / 12 ; 2$ semantic paraphasias); and poor constitution (3/8). His Frontal Assessment Battery (FAB) score of $7 / 18$ revealed poor performance on the subtests of similarity $(1 / 3)$, verbal fluency $(0 / 3)$, motor series $(1 / 3)$, conflicting instructions (1/3), and inhibitory control (1/3). The performance measures on the Stroop test for word and color identification were $33.28 \mathrm{~s}$ with 1 error for part 1 , and 128.7 $\mathrm{s}$ with 10 errors for part 2 . The difference of $95.46 \mathrm{~s}$ between parts 1 and 2 was considerably greater than normal (< $11.5 \mathrm{~s})$.

\section{Discussion}

NIID is clinically heterogeneous. A previous report proposed three clinical subgroups of NIID: infantile, juvenile, and adult-onset forms (12). A meaningful neuropsychological examination is difficult in infantile and juvenile NIID due to the short clinical course. Adult-onset NIID is characterized by memory loss; cognitive dysfunction and disorientation with or without extrapyramidal signs; cerebellar ataxia; involuntary movements; and autonomic dysfunction $(12,15)$. We previously reported that a skin biopsy was useful for the diagnosis of NIID (16). In NIID skin biopsy samples, intranuclear inclusions have been observed in adipocytes, fibroblasts, and sweat gland cells, without structural abnormalities (16). Head MRI is also useful for the diagnosis of NIID. The finding of a high signal intensity in the corticomedullary junction on DWI is characteristic of NIID (Figure B) (18).

The present patient presented with symptoms of dementia; left-sided cerebellar ataxia; hyporeflexia and hypopallesthesia. He also had autonomic dysfunctions involving miosis, neurogenic bladder, and orthostatic hypotension. FLAIR images showed leukoencephalopathy (Figure A), and DWI showed hyperintensity of the corticomedullary junction (Figure B). Subsequently, we diagnosed our patient as having adult-onset NIID by a skin biopsy combined with these MRI findings (Figure A, B, D, F) (16).

Although adult-onset NIID patients reportedly show signs and symptoms of dementia, sufficient objective neuropsychological assessments have not been performed in these studies $(3,6,9,12,18)$. In our patient, whose condition was diagnosed from a skin biopsy, we were able to assess a higher brain function. Neuropsychological testing revealed memory loss, visuospatial dysfunction, and frontal cognitive dysfunction, especially in language and executive functions. The ACE-R is a brief battery of tests for six cognitive domains (orientation, attention, memory, verbal fluency, language, and visuospatial ability) and, similar to the MMSE, is useful for detecting dementia (19). Remote memory and disorientation were impaired equally on the MMSE and ACE-R, although the performance of the MMSE was inconsistent with that of the ACE-R in terms of recent memory indicators (e.g., delayed recall). This might have been due to the patient's difficulty in concentrating. Verbal fluency was markedly impaired on the ACE-R and FAB. This result is similar to that of a previous study, in which the verbal output in patients with adult-onset NIID was characterized by impaired word finding, verbal paraphasias, and perseveration (6). The visuospatial function, as assessed by the MMSE and ACE-R, was also impaired in our patient-a finding not described in previous case reports $(3,6,9,18)$. The patient's performance on the FAB and Stroop tests clearly demonstrated frontal executive dysfunction. Abnormal behaviors described in previous reports, such as repetitive outbursts of violence (6), wandering aimlessly (9), and absentmindedly standing naked (18), were not observed in our patient. Previous studies have reported that intranuclear inclusion bodies were found frequently in glial cells and were abundantly present in the association cortices of the frontal lobe $(6,9,12)$; these features may be associated with frontal cognitive dysfunction. In our patient, the frontal-dominant white matter lesions observed on MRI and the frontoparietal hypoperfusion observed on SPECT of the head (Figure $\mathrm{A}, \mathrm{C}$ ) were consistent with the results of frontal cognitive dysfunction.

Generally, adult-onset genetic leukoencephalopathy is best known as cerebral autosomal dominant arteriopathy with subcortical infarcts and leukoencephalopathy (CADASIL) (20). CADASIL is a good model for subcortical ischemic strokes and pure vascular dementia (21). As in our NIID patient, executive dysfunction and deficits in attention or concentration, visuospatial skills, language, and verbal or visual memory have been described as common manifestations of CADASIL and vascular dementia (20-22). Although reports on the pathology of NIID have shown no atheroscle- 
rotic disease $(6,9)$, these similarities suggest that adult-onset NIID might have a pathological mechanism similar to that of leukoencephalopathy.

The mechanisms of nuclear inclusion formation in NIID are unknown $(7,15)$. As in NIID, nuclear inclusions have been described in polyglutamine (polyQ) diseases, including Huntington's disease, dentatorubropallidoluysian atrophy (DRPLA), spinobulbar muscular atrophy, and spinocerebellar ataxias 1, 2, 3, 6, 7, and $17(15,23)$. PolyQ diseases share a pathogenetic mechanism, namely the expansion of a translated CAG repeat (15), whereas the pathogenetic mechanism in NIID is uncertain. Pathologically, the localization of inclusions differs between NIID and polyQ diseases; in NIID there are neuronal and glial intranuclear inclusions, whereas in polyQ diseases there are neuronal and glial inclusions as well as both intranuclear and cytoplasmic inclusions $(1-15,23,24)$. The intranuclear inclusions in NIID are distributed in the neurons of the brain, spinal cord, dorsal root, sympathetic ganglia, and peripheral nerves $(1-12,15)$. In contrast, polyQ diseases show neuronal degeneration with nuclear inclusions specifically in the brainstem and cerebellar efferent pathways, consistent with the clinical features (15). Clinically, depending on the age of onset and the clinical phenotype, the differential diagnoses in polyQ diseases can include DRPLA (24), whereas signs of ataxia in DRPLA are more dominant than those in NIID (24), NIID exhibits multi-systemic degeneration involving the central, peripheral, and autonomic nervous systems (1-12, 15). In terms of higher brain dysfunction, the frontal lobe dysfunction in our NIID patient may be similar to those of vascular dementia rather than the recent and episodic memory loss characterized in Alzheimer's disease, the most common form of dementia (25).

The presence of a high signal intensity at the corticomedullary junction on DWI with leukoencephalopathy would then strongly suggest that a skin biopsy is required. Our case suggests that adult-onset NIID patients may show an impaired language and executive function. However, a greater accumulation of cases is needed to verify the cognitive dysfunction in NIID patients.

The authors state that they have no Conflict of Interest (COI).

\section{References}

1. Sung JH, Ramirez-Lassepas M, Mastri AR, et al. An unusual degenerative disorder of neurons associated with a novel intranuclear hyaline inclusion (neuronal intranuclear hyaline inclusion disease). A clinicopathological study of a case. J Neuropathol Exp Neurol 39: 107-130, 1980 .

2. Lindenberg R, Rubinstein LJ, Herman MM, Haydon GB. A light and electron microscopy study of an unusual widespread nuclear inclusion body disease. A possible residuum of an old herpesvirus infection. Acta Neuropathol 10: 54-73, 1968.

3. Schuffler MD, Bird TD, Sumi SM, Cook A. A familial neuronal disease presenting as intestinal pseudoobstruction. Gastroenterology 75: 889-898, 1978.

4. Patel H, Norman MG, Perry TL, Berry KE. Multiple system atrophy with neuronal intranuclear hyaline inclusions. Report of a case and review of the literature. J Neurol Sci 67: 57-65, 1985.

5. Soffer D. Neuronal intranuclear hyaline inclusion disease presenting as Friedreich's ataxia. Acta Neuropathol 65: 322-329, 1985.

6. Munoz-Garcia D, Ludwin SK. Adult-onset neuronal intranuclear hyaline inclusion disease. Neurology 36: 785-790, 1986.

7. Funata N, Maeda Y, Koike M, et al. Neuronal intranuclear hyaline inclusion disease: report of a case and review of the literature. Clin Neuropathol 9: 89-96, 1990.

8. Barnett JL, McDonnell WM, Appelman HD, Dobbins WO. Familial visceral neuropathy with neuronal intranuclear inclusions: diagnosis by rectal biopsy. Gastroenterology 102: 684-691, 1992.

9. Weidenheim KM, Dickson DW. Intranuclear inclusion bodies in an elderly demented woman: a form of intranuclear inclusion body disease. Clin Neuropathol 14: 93-99, 1995.

10. O'Sullivan JD, Hanagasi HA, Daniel SE, Tidswell P, Davies SW, Lees AJ. Neuronal intranuclear inclusion disease and juvenile parkinsonism. Mov Disord 15: 990-995, 2000.

11. Zannolli R, Gilman S, Rossi S, et al. Hereditary neuronal intranuclear inclusion disease with autonomic failure and cerebellar degeneration. Arch Neurol 59: 1319-1326, 2002.

12. Takahashi-Fujigasaki J. Neuronal intranuclear hyaline inclusion disease. Neuropathology 23: 351-359, 2003.

13. Kulikova-Schupak R, Knupp KG, Pascual JM, Chin SS, Kairam $\mathrm{R}$, Patterson MC. Rectal biopsy in the diagnosis of neuronal intranuclear hyaline inclusion disease. J Child Neurol 19: 59-62, 2004.

14. Sone J, Hishikawa $\mathrm{N}$, Koike $\mathrm{H}$, et al. Neuronal intranuclear hyaline inclusion disease showing motor-sensory and autonomic neuropathy. Neurology 65: 1538-1543, 2005.

15. Woulfe JM. Abnormalities of the nucleus and nuclear inclusions in neurodegenerative disease: a work in progress. Neuropathol Appl Neurobiol 33: 2-42, 2007.

16. Sone J, Tanaka F, Koike H, et al. Skin biopsy is useful for the antemortem diagnosis of neuronal intranuclear inclusion disease. Neurology 76: 1372-1376, 2011.

17. Kitagawa N, Sone J, Sobue G, Kuroda M, Sakurai M. Neuronal intranuclear inclusion disease presenting with resting tremor. Case Rep Neurol 6: 176-180, 2014.

18. Sone J, Kitagawa N, Sugawara E, et al. Neuronal intranuclear inclusion disease cases with leukoencephalopathy diagnosed via skin biopsy. J Neurol Neurosurg Psychiatry 85: 354-356, 2014.

19. Miyoshi E, Dawson K, Mitchell J, Arnold R, Hodges JR. The Addenbrooke's Cognitive Examination Revised (ACE-R): a brief cognitive test battery for dementia screening. Int $\mathrm{J}$ Geriatr Psychiatry 21: 1078-1085, 2006.

20. Di Donato I, Banchi S, Federico A, Dotti MT. Adult-onset genetic leukoencephalopathies. Focus on the more recently defined forms. Curr Mol Med 2014 (in press).

21. Chabriat H, Joutel A, Dichgans M, Tournier-Lasserve E, Bousser MG. Cadasil. Lancet Neurol 8: 643-653, 2009.

22. Moorhouse P, Rockwood K. Vascular cognitive impairment: current concepts and clinical developments. Lancet Neurol 7: 246255, 2008.

23. Holmberg M, Duyckaerts C, Dürr A, et al. Spinocerebellar ataxia type 7 (SCA7): a neurodegenerative disorder with neuronal intranuclear inclusions. Hum Mol Genet 7: 913-918, 1998.

24. Hayashi Y, Kakita A, Yamada M, et al. Hereditary dentatorubralpallidoluysian atrophy: detection of widespread ubiquitinated neuronal and glial intranuclear inclusions in the brain. Acta Neuropathol 96: 547-552, 1998.

25. McKhann GM, Knopman DS, Chertkow H, et al. The diagnosis of dementia due to Alzheimer's disease: recommendations from the National Institute on Aging-Alzheimer's Association workgroups on diagnostic guidelines for Alzheimer's disease. Alzheimers Dement 7: 263-269, 2011.

(C) 2016 The Japanese Society of Internal Medicine

http://www.naika.or.jp/imonline/index.html 